\title{
Leptospirosis: clinical aspects
}

\author{
Author: Senaka Rajapakse ${ }^{\mathrm{A}}$
}

Leptospirosis is one of the most important zoonotic bacterial diseases worldwide, commonly affecting resource-poor populations and resulting in significant morbidity and mortality. This article provides an overview of the epidemiology, clinical manifestations, diagnosis and treatment of human leptospirosis.

\section{Introduction}

Leptospirosis is one of the most important zoonotic bacterial infections worldwide., ${ }^{1,2}$ It most commonly affects resource-poor populations, resulting in significant morbidity and many deaths. The infection is estimated to cause one million cases and around 58,900 deaths annually, with a case fatality ratio of $6.85 \% .^{3}$

\section{History}

Adolph Weil first described leptospirosis in 1886 as a febrile illness with icterus, enlarged spleen, renal failure and conjunctivitis associated with outdoor occupations where people came into contact with water. ${ }^{4}$ Thus, the severe form was named 'Weil's disease'. There are several descriptions reported much earlier in ancient texts that match the clinical features of leptospirosis: 'cane-cutter's disease' or 'swineherd's disease' in Europe, 'rice field jaundice' in ancient Chinese texts, and 'Akiyami (autumn fever)' in Japan. The causative organism was first described in 1907 by Stimson, who demonstrated the presence of spirochaetes in the kidneys of a patient dying of the disease; the organism was named Spirochaeta interrogans because of the question-mark shape of the organism. ${ }^{1}$

\section{Taxonomy and classification}

Leptospirae are free-living aerobic spirochaetes with characteristic hooked ends, $6-20 \mu \mathrm{m}$ in length, with an approximate diameter of $0.1 \mu \mathrm{m}$. The surface structures of leptospirae are composed of a cytoplasmic membrane and a peptidoglycan outer cell wall, and an outer membrane sheath composed of a lipid bilayer, ${ }^{5}$ thus showing features of both gram-negative and gram-positive bacteria. The organisms are motile, by means of endoflagellae.

Only certain strains cause disease in mammalian hosts. The taxonomy is complex. ${ }^{6}$ Traditionally, the genus Leptospira was divided into two species: $L$ interrogans (all the pathogenic strains) and $L$ biflexa (all the saprophytic strains). Currently, leptospira are

Author: ${ }^{\text {A }}$ rofessor in clinical medicine, University of Colombo, Colombo, Sri Lanka classified according to genotypes, and according to serovars. There are 21 species based on genotypic classification, nine of which are pathogenic, and five intermediate, the rest being non-pathogenic strains. Most of the pathogenic strains are classified under the $L$ interrogans block. Serovars and serogroups are classified based on serological testing, and not DNA. Around 240 serovars have been identified, and these are grouped into several serogroups. Only a few of these are pathogenic. Strains with similar DNA can appear in different serogroups, and serovars belonging to the same serogroup can be genetically diverse. In the currently accepted taxonomy, the nomenclature is as follows: 'Genus species serovar Serovar name' for example, Leptopira interrogans serovar Ballum.

\section{Epidemiology and transmission}

Leptospirosis is highly prevalent in the tropics, with $73 \%$ of cases occurring in this zone, in particular in South-East Asia, East

\section{Key points}

Leptospirosis is a zoonosis which is highly prevalent in the tropics. Transmission is primarily through occupational exposure to rodents and other animals, although recreational exposure is also known to occur.

Acute kidney injury, hepatic derangement, myocardial involvement and pulmonary haemorrhage are serious complications, and a myriad of other manifestations have been reported.

Diagnosis of leptospirosis is based on characteristic clinical features occurring with a history of risk exposure, together with laboratory confirmation through serology or detection of the organism or its DNA.

Antibiotics and supportive therapy are the mainstay of management. No other specific evidence-based therapies exist at present.

No effective vaccine is currently available, and prevention is through avoidance of risk exposure, with a degree of protection provided by chemoprophylaxis.

KEYWORDS: leptospirosis, Leptospira, acute febrile illness, jaundice, pulmonary haemorrhage DOI: 10.7861/clinmed.2021-0784 
Sub-Saharan Africa, the Caribbean and Oceania. It is common among the rural farming populations and impoverished urban and semi-urban populations, particularly affecting young male adults. Farmers, those in contact with livestock, those exposed to rodents at their workplace, and people living in areas where sanitation is poor are at highest risk. ${ }^{3}$ Recreational exposure in those undertaking water sports has also been described. ${ }^{7}$ Urban outbreaks are common in settings where sanitation is poor, and rats breed in abundance. ${ }^{8}$ Incidence rates have generally been stable globally; however, numerous large outbreaks have occurred from time to time in certain countries, in some instances related to natural disasters associated with flooding. ${ }^{3}$

Nearly all mammals can serve as carriers of leptospirosis, harbouring and shedding the organisms from the proximal tubules of the kidneys. ${ }^{2}$ The rat is by far the most important carrier responsible for human leptospirosis. This is because of the ubiquitous presence of rats in the proximity of human habitats, and because rats excrete very high concentrations of organisms, even several months after initial infection. The commonest means of transmission to humans is through abrasions on the skin and mucous membranes coming into contact with water contaminated with infected rat urine. Humans are incidental hosts, and are at greater risk when working or living in environments in proximity with the maintenance hosts, especially rats and farm animals. Effective control of leptospirosis is difficult because of two epidemiological features of the disease. Firstly, leptospira are capable of developing a symbiotic relationship with many animal hosts, where the organisms persist in the renal tubules for long periods, with shedding of the bacteria in the urine, without causing disease to the host. Secondly, wild animals provide an important reservoir, continuously re-infecting domestic animals.

\section{Clinical manifestations}

Human leptospirosis has diverse clinical manifestations. Clinical illness in humans can range from a mild, self-limiting acute febrile illness to a severe, life-threatening condition with multiple organ dysfunction. ${ }^{1,2}$ Many organ systems can be involved, to varying degrees, and an array of atypical or unusual manifestations and complications have also been described. The clinical features of leptospirosis are similar to those seen in many other febrile illnesses, especially diseases seen in the tropics, such as dengue and other haemorrhagic fevers, rickettsial infection, malaria, and bacterial sepsis. While the majority present with uncomplicated fever, approximately $10 \%$ develop severe disease. ${ }^{2,9}$ The classical presentation with conjunctival suffusion, jaundice, and acute kidney injury constitutes Weil's syndrome. ${ }^{2}$ Pulmonary haemorrhage has recently been shown to be an important cause of mortality. ${ }^{2,10}$

The incubation period shows wide variation, from 2-20 days, usually $7-12$ days. A biphasic illness is seen in some patients. The clinical course of leptospirosis has been classically divided into a 'leptospiraemic phase' or acute phase, followed by an 'immune phase.'11 The initial 'leptospiraemic phase' is said to last for three to nine days, and presents as a non-specific acute febrile illness. Fever, chills, myalgia and headache are present. Conjunctival suffusion is a characteristic finding, developing on the third to fourth day. Myalgia can be severe, and can usually involves the calf, abdomen (mimicking acute abdomen) and paraspinal muscles (resulting in meningism). The 'leptospiraemic' or 'septicaemic' phase is followed by an immune phase, where IgM antibodies appear in the blood, and organisms are excreted in the urine. Presumably, the organisms settle in higher concentrations in the proximal tubules of the kidney (and other organs) during this phase. Depending on the degree of organ involvement and the virulence of the organism, serious manifestations occur during this stage. In practice, this differentiation into phases is arbitrary, and while there can be a brief period of defervescence between these phases, there often is overlap between them. While recovery takes place in the majority of patients, in a small number of infected individuals there is persistent high fever, with the development of jaundice, acute kidney injury and other serious organ dysfunction.

The pathogenesis of severe leptospirosis is poorly understood; however, it is thought to be due to a form of vasculitis. As with other bacterial infections, both direct damage to tissues by leptospira and immune-mediated mechanisms are responsible for tissue and organ damage, deranged tissue microcirculation and endothelial dysfunction. Although jaundice is a prominent feature, death often results from complications of acute kidney injury, myocardial involvement or pulmonary haemorrhage. Pulmonary involvement, in extreme forms with pulmonary haemorrhage, other haemorrhagic manifestations, and myocarditis are important, serious manifestations resulting in high mortality. A myriad of atypical or unusual manifestations have been reported with human leptospirosis, including nervous system involvement (acute disseminated encephalomyelitis, hydrocephalus and raised intracranial pressure, encephalitis-induced coma, intracranial vascular events; intracranial bleeding and thrombosis, cerebellar syndrome, transverse myelitis, Guillain-Barré syndrome, mononeuritis and mononeuritis multiplex including cranial nerve palsies), ocular manifestations (uveitis, optic neuritis, retinal phlebitis), haematological involvement (pancytopaenia, haemolytic anaemia, hemolytic uremic syndrome and thrombotic thrombocytopenic purpura) and gastrointestinal involvement (pancreatitis, cholecystitis). $^{12}$

\section{Diagnosis}

Diagnosis of leptospirosis is made largely on the presence of suggestive clinical features, with a history of risk exposure. Leptospirosis should be suspected in any patient with a history of risk exposure, and any of the following: headache, myalgia, prostration, jaundice, conjunctival suffusion, oliguria, features of meningeal irritation, haemorrhage, features of cardiac failure or arrhythmia, cough, breathlessness, skin rash, or any other evidence of organ involvement or dysfunction (Box 1).

Many diagnostic tests are available for leptospirosis, but the availability of such tests in resource-poor settings is limited. Diagnostic accuracy is also variable, especially for serological tests. The cut-off values for seropositivity in a single sample are likely to depend on the regional seroprevalence. Cross-reactivity with other infections can further confound the results.

Broadly, the diagnostic tests are divided into those which provide direct evidence of infection (demonstration of leptospires or its DNA, or culture) and tests providing indirect evidence of infection (demonstration of antibodies to leptospirosis) (Box 2).

\section{Management}

Patients with suspected or confirmed leptospirosis with mild clinical features and no comorbidities may be managed as outpatients with regular follow up for identification of complications. ${ }^{13}$ Patients who have clinical features of organ involvement, or those who 


\section{Box 1. Features of organ involvement in leptospirosis}

\section{Renal involvement}

$>$ Reduced urine output

Haematuria, granular casts or red cell casts in the urine

Acute kidney injury (any degree)

\section{Liver involvement}

$>$ Jaundice

$>$ Tender hepatomegaly

$>$ Rise in serum aspartate transaminase or alanine transaminase three times above the upper limit of normal

> Raised serum bilirubin, alkaline phosphatase or gamma glutamyl transpeptidase

$>$ Prolonged prothrombin time

\section{Pulmonary involvement}

$>$ Cough, breathlessness, haemoptysis

Hypoxia-oxygen saturation $<94 \%$

Respiratory rate $>30 / \mathrm{min}$

$>$ Crackles and wheezes on auscultation

$>$ Evidence of lung parenchymal involvement on chest radiography or $\mathrm{CT}$ scan

$>$ Acute respiratory distress syndrome

\section{Cardiac involvement}

> Shortness of breath, chest pain, palpitations, tachycardia, basal crackles

$>$ Hypotension

> ECG abnormalities: arrhythmias, ST/T changes, conduction abnormalities

> Wall motion abnormalities on echocardiography

\section{Haematological involvement}

$>$ Bleeding manifestations

$>$ Thrombocytopaenia $<130 \times 10^{9} / \mathrm{L}$

Deranged coagulation

Disseminated intravascular coagulopathy

\section{Neurological involvement}

Reduced level of consciousness

Meningism

Focal neurological signs

have comorbidities, must be admitted for in-hospital care. Early initiation of antibiotic treatment is likely to improve outcome.

For mild disease (ie without organ involvement):

> Administer doxycycline 100 mg twice daily for 7 days.

$>$ Do the following investigations: complete blood count, C-reactive protein, creatinine, urea, electrolytes, liver transaminases.

$>$ bilirubin, urine full report.

$>$ Monitor urine output.

$>$ Review every 48 hours.

$>$ Admit to hospital if there is jaundice, reduction in urine output, haematuria, cough, or difficulty breathing, or if clinically very ill.

For those requiring hospital admission:

> Do the following investigations: complete blood count, C-reactive protein, creatinine, urea, electrolytes, liver transaminases, bilirubin, urine full report, coagulation tests and blood picture (to identify disseminated intravascular coagulopathy), ECG, chest radiograph.
Box 2. Diagnostic tests for leptospirosis

\section{Isolation of leptospires}

> Blood culture for leptospirosis: gold standard test, requires samples during the first week of illness. Labour intensive and time consuming, requires high levels of biosafety levels, low diagnostic yield. Used for identification of serovar, and for determining antibiotic sensitivity.

$>$ Polymerase-chain reaction (PCR): useful for early diagnosis (first week of acute illness). High sensitivity and specificity. Useful for genomic classification.

> Dark-ground microscopy of body fluids: low sensitivity, obsolete now.

\section{Serological methods}

Antibodies are usually detectable by day 6-10 of illness and peak within 3-4 weeks. Comparison of acute and convalescent have high sensitivity and specificity. Can be used for genus or serogroup identification.

> Microscopic agglutination test: serological reference standard. However, requires experienced observers to reduce inter-observer variation. Time consuming and hazardous as live cultures are required to provide antigen. Available only in reference laboratories.

> IgM enzyme-linked immunosorbent assay (ELISA): easily available. Sensitivity and specificity depend on regional patterns of seropositivity.

$>$ Commence intravenous antibiotics: penicillin G 1.5 million units 6 hourly, or ceftriaxone $1 \mathrm{~g}$ twice daily for 7 days. For those with penicillin or cephalosporin allergy, doxycycline or a macrolide (azithromycin or clarithromycin) may be used.

$>$ Monitor fluid intake and urine output.

> Fluid intake should be guided by clinical assessment of hydration. In adults, the intake should be around $2.0-2.5 \mathrm{~L}$ per 24 hours.

> If oliguric, the daily intake should be equal to the previous day's urine output plus estimated insensible loss (usually around $500 \mathrm{ml}$ ). If the previous day's output is not known, the hourly intake should be calculated as the previous hour's urine output plus $25 \mathrm{ml}$.

> All nephrotoxic and hepatotoxic drugs should be stopped. Anticoagulants and antiplatelet drugs may need to be stopped if bleeding manifestations are present.

> Critically ill patients (ie those with haemodynamic instability, respiratory compromise, haemoptysis, reduced consciousness, or other signs of organ dysfunction) will require to be managed in a high dependency unit or intensive care unit.

$>$ Those requiring intensive care should be managed in accordance with standard critical care management protocols and guidelines.

> Mechanical ventilatory support may be required, especially in pulmonary haemorrhage and acute respiratory distress syndrome.

$>$ The use of high dose corticosteroids for treatment of leptospirosis is not supported by high quality evidence, and routine use is not recommended. There are reports of potential adjunctive benefit with high dose corticosteroids in severely ill patients.

> Plasmapheresis has been used in severe leptospirosis, with some non-randomised trials showing benefit, but the evidence is of low quality. 
Supportive care and antibiotics remain the mainstay of management in leptospirosis, and there are as yet no specific therapies which influence outcome.

\section{Prevention}

Prevention of leptospirosis is through avoidance of potential exposure to infection, and administration of pharmacological prophylaxis to individuals at high risk. Doxycycline $200 \mathrm{mg}$ weekly, commencing one week prior to exposure, and continued through the period of exposure is recommended. No human vaccine is currently available.

\section{Future directions}

There are currently no reliable scoring systems or predictive models to determine which patients with leptospirosis are likely to develop severe disease; this is an area for future research. The pathogenesis of severe leptospirosis is poorly understood, and basic sciences research should focus on identifying biomarkers of severity, and potential therapeutic targets. Further randomised trials to evaluate potentially beneficial interventions which could prevent progression to severe disease are required. ${ }^{14}$

\section{References}

1 Levett PN. Leptospirosis. Clin Microbiol Rev 2001;14:296-326.

2 Haake DA, Levett PN. Leptospirosis in humans. Curr Top Microbiol Immunol 2015;387:65-97.

3 Costa F, Hagan JE, Calcagno ] et al. Global morbidity and mortality of leptospirosis: A systematic review. PLoS Negl Trop Dis 2015;9:e0003898.

4 Weil A. Ueber einer eigenhuemliche, mit Milztumor, Icterus un Nephritis einhergehende, acute Infektionskrankheit. Deutsch Arch Klin Med 1886;39:109.
5 Evangelista KV, Coburn J. Leptospira as an emerging pathogen: a review of its biology, pathogenesis and host immune responses. Future Microbiol 2010;5:1413-25.

6 Davies HD, Simonsen KA Leptospira. In: Kliegman RM, ed. Nelson Textbook of Pediatrics, 21 ed. Elsevier, 2020:1601-3.

7 Agampodi SB, Karunarathna D, Jayathilala $\mathrm{N}$ et al. Outbreak of leptospirosis after white-water rafting: sign of a shift from rural to recreational leptospirosis in Sri Lanka? Epidemiol Infect 2014;142:843-6.

8 Felzemburgh RD, Ribeiro GS, Costa F et al. Prospective study of lep tospirosis transmission in an urban slum community: role of poor environment in repeated exposures to the Leptospira agent. PLOS Negl Trop Dis 2014;8:e2927.

9 Adler B, de la Pena Moctezuma A. Leptospira and leptospirosis. Vet Microbiol 2010;140:287-96.

10 Trevejo RT, Rigau-Perez JG, Ashford DA et al. Epidemic leptospirosis associated with pulmonary hemorrhage - Nicaragua, 1995. J Infect Dis 1998;178:1457-63.

11 Turner LH. Leptospirosis I. Trans R Soc Trop Med Hyg 1967:61:842-55.

12 Rajapakse S, Rodrigo C, Balaji K, Fernando SD. Atypical manifestations of leptospirosis. Trans R Soc Trop Med Hyg 2015;109:294-302.

13 Ministry of Health, Nutrition and Indigenous Medicine Sri Lanka. National guidelines on management of leptospirosis. MoH, 2016. www.epid.gov.lk/web/images/pdf/Publication/leptospirosis/lepto_ national_guidelines.pdf [Accessed 21 Dcember 2021].

14 Rajapakse S, Rodrigo C, Handunnetti SM, Fernando SD. Current immunological and molecular tools for leptospirosis: diagnostics, vaccine design, and biomarkers for predicting severity. Ann Clin Microbiol Antimicrob 2015;14:2.

Address for correspondence: Professor Senaka Rajapakse, Department of Clinical Medicine, Faculty of Medicine, University of Colombo, 25, Kynsey Road, Colombo 08, Sri Lanka. Email: senaka@med.cmb.ac.lk 